\title{
THE FOUR-VERTEX THEOREM FOR A CERTAIN TYPE OF SPACE CURVES.*
}

\author{
BY W. C. GRAUSTEIN AND S. B. JACKSON
}

It is the purpose of this note to establish for a certain type, $A$, of space curves the new form of the four-vertex theorem recently stated and proved for plane ovals. $\dagger$

A space curve $C$ shall be said to be of type $A$ provided (a) it is a closed regular curve of class $C^{\prime \prime}$, (b) its curvature never vanishes, (c) the projection of its tangent indicatrix $I$ on a plane $\pi$ perpendicular to the line joining the origin $O$ to the center of gravity $G$ of $I$ is an oval or, if $G$ coincides with $O$, the projection of $I$ on some plane, $\pi$, is an oval, and (d) this oval is traced just once when $C$ is traced once.

By a vertex shall be meant a point, or an arc of constant curvature, for which the curvature has a relative extremum with respect to the neighboring arcs on either side. A vertex shall be said to be primary if the curvature at it has a maximum (minimum) which is greater (less) than the average curvature of the curve with respect to the arc. Otherwise, a vertex shall be termed secondary. $\ddagger$

The theorem to be established may now be formulated as follows.

Statement I. On a curve of type A, whose curvature is not constant, there are at least four primary vertices. More precisely, the number of primary vertices, if finite, exceeds the number of secondary vertices by at least four, and is infinite if the number of secondary vertices is infinite.

* Presented to the Society, March 26, 1937.

$\dagger$ W. C. Graustein, $A$ new form of the four-vertex theorem, Monatshefte für Mathematik und Physik, vol. 43 (1936), pp. 381-384; for a related theorem, see Hayashi, Some general applications of Fourier series, Rendiconti del Circolo Matematico di Palermo, vol. 50 (1926), p. 100. For other work on the fourvertex theorem in space see Süss, Ein Vierscheitelsatz bei geschlossenen Raumkurven, Tôhoku Mathematical Journal, vol. 29 (1928), pp. 359-362; Takasu, Vierscheitelsatz filr Raumkurven, Tôhoku Mathematical Journal, vol. 39 (1934), pp. 292-298, and vol. 41 (1936), pp. 317, 318.

$\ddagger$ Ibid., p. 381 . 
The theorem may be stated more simply in terms of the concept of a transition of the curvature.* If $1 / R$ is the curvature of the curve and $1 / A$ the average curvature with respect to the arc, a transition of the curvature is defined as a point, or arc, of the curve for which $1 / R$ has the value $1 / A$ and $1 / R-1 / A$ changes sign, that is, has opposite signs for neighboring arcs on either side.

A transition of the curvature is never a vertex, by definition. Moreover, from the continuity of $1 / R-1 / A$ it follows that the number of primary vertices between two consecutive transitions of the curvature exceeds the number of secondary vertices on this arc by exactly one, or both types of vertices are infinite in number. $\uparrow$ Hence our theorem may be restated as follows:

Statement II. On a curve of type $A$, whose curvature is not constant, there are at least four transitions of the curvature.

In the proof of the theorem we shall prefer to deal with the radius of curvature of the given curve $C$ rather than with the curvature and to employ, as parameter, not the arc $s$ of $C$, but the $\operatorname{arc} \phi$ of the tangent indicatrix $I$. If $\phi$ is measured in the direction of increasing $s$, we have

$$
\frac{1}{R}=\frac{d \phi}{d s} .
$$

Hence, since $1 / R>0, \phi$ is an admissible parameter for both $C$ and $I$. Furthermore, since $1 / R$ is a continuous function of $s$ which is periodic of period $D$-the length of $C, R$ is a singlevalued continuous function of $\phi$ which is periodic of period $d-$ the length of $I$.

From the relations $d s=R d \phi$ and $d \phi=d s / R$, it follows that

$$
\int_{0}^{d} R d \phi=D, \quad \int_{0}^{D} \frac{d s}{R}=d .
$$

* Ibid., p. 382.

$\dagger$ This statement may be expressed quantitatively by the equation

$$
P=S+T
$$

where $P, S$, and $T$ denote, respectively, the number of primary vertices, secondary vertices, and transitions of curvature, and where, furthermore, it is understood that if $S$ or $T$ is infinite the equation is simply to mean that $P$ is infinite. 
Consequently, the average radius of curvature of $C$ with respect to the arc $\phi$ of $I$, namely $A=D / d$, is the reciprocal of the average curvature of $C$ with respect to the arc $s$ of $C$, namely, $1 / A=d / D$. Thus the definitions of primary and secondary vertices and that of a transition of the curvature might just as well have been based on comparisons of the radius of curvature with the average radius of curvature with respect to $\phi$.

The tangent indicatrix, $I$, of the curve $C$ has the parametric equations

$$
y_{i}=\alpha_{i}(\phi), \quad(i=1,2,3),
$$

where $\alpha_{1}, \alpha_{2}, \alpha_{3}$ are the direction cosines of the directed tangent to $C$ at the point $\phi=\phi$. For $C$ itself we have the equations

$$
d x_{i}=R \alpha_{i} d \phi, \quad(i=1,2,3),
$$

with the conditions of closure

$$
\int_{0}^{d} R \alpha_{i} d \phi=0, \quad(i=1,2,3) .
$$

These conditions, together with the fact that $A$ is the average value of $R$ with respect to $\phi$, may be written in the forms

$$
\int_{0}^{d}(R-A) d \phi=0, \quad \int_{0}^{d}(R-A) \alpha_{i} d \phi=-D \bar{\alpha}_{i}, \quad(i=1,2,3),
$$

where $\left(\bar{\alpha}_{1}, \bar{\alpha}_{2}, \bar{\alpha}_{3}\right)$ are the coördinates of the center of gravity, $G$, of the curve $I$, and hence in the form of the identity

$$
\begin{aligned}
\int_{0}^{d}\left(a_{0}+a_{1} \alpha_{1}+a_{2} \alpha_{2}+a_{3} \alpha_{3}\right)(R & -A) d \phi \\
& \equiv-D\left(a_{1} \bar{\alpha}_{1}+a_{2} \bar{\alpha}_{2}+a_{3} \bar{\alpha}_{3}\right)
\end{aligned}
$$

in the arbitrary constants $a_{0}, a_{1}, a_{2}, a_{3}$.

The right-hand side of this identity vanishes if the direction with the components $a_{1}, a_{2}, a_{3}$ is perpendicular to the line $O G$ or if, as in the case of a plane oval, $G$ coincides with $O$. Thus, we pass to the following conclusion.

LEMMA 1. The relation

$$
\int_{0}^{d}\left(a_{0}+a_{1} \alpha_{1}+a_{2} \alpha_{2}+a_{3} \alpha_{3}\right)(R-A) d \phi=0
$$


holds for every plane $a_{0}+a_{1} x_{1}+a_{2} x_{2}+a_{3} x_{3}=0$ which is perpendicular to the plane $\pi$ associated with the given curve $C$ of type $A$ after the manner described in the definition of a curve of this type.

We may now establish our theorem, by assuming that it is false. There are, then, since $R \neq \equiv A$, just two transitions of the curvature. In other words, there exist two points $P, Q$ on $I$ such that $R-A$ is of one sign or zero for one of the arcs, $P Q$, into which the points divide $I$ and is of the opposite sign or zero for the other arc, $Q P$.

Consider, next, the function $a_{0}+a_{1} \alpha_{1}+a_{2} \alpha_{2}+a_{3} \alpha_{3}$, where the $a$ 's are so chosen that $a_{0}+a_{1} x_{1}+a_{2} x_{2}+a_{3} x_{3}=0$ represents the plane through $P$ and $Q$ which is perpendicular to $\pi$. This plane cuts the oval which is the projection of $I$ on $\pi$ in just two points, which are necessarily the projections of $P$ and $Q$, and of $P$ and $Q$ only. Hence, it divides the oval into two arcs, one on either side of it, and these arcs must be the projections of the arcs $P Q$ and $Q P$ of $I$. It follows, then, that the function in question is positive or zero for one of the latter arcs, and negative or zero for the other.

It is now evident that the expression

$$
\left(a_{0}+a_{1} \alpha_{1}+a_{2} \alpha_{2}+a_{3} \alpha_{3}\right)(R-A),
$$

never changes sign, and so the integral of it extended around $I$ cannot be zero. But this contradicts Lemma 1 and our theorem is established.

The reader may observe that, when the center of gravity of $I$ is at the origin, it is unnecessary to assume that there exists a plane on which the projection of $I$ is an oval. It is sufficient to make the less restrictive assumption that through each two points of $I$ there passes a plane, $p$, dividing $I$ into exactly two arcs, one on either side of $p$. This follows from the fact that, when $\bar{\alpha}_{i}=0, i=1,2,3$, Lemma 1 holds for any plane whatever.

The existence of twisted curves of type $A$ is readily established. Fujiwara has shown that a closed curve on the unit sphere is the tangent indicatrix of a closed space curve if and only if the center, $O$, of the sphere lies interior to the convex hull of the curve.* The problem of exhibiting a twisted space

* M. Fujiwara, Über die kleinsten eine Kurve enthaltenden konvexen Körper, The Science Reports of the Tôhoku Imperial University, vol. 4 (1915), p. 341. 
curve of type $A$ is thus reduced to that of finding a spherical curve, other than a great circle, whose convex hull has $O$ as an interior point and which projects into an oval on a properly chosen plane.

On the unit sphere $x_{1}=\sin \phi \cos \theta, x_{2}=\sin \phi \sin \theta, x_{3}=\cos \phi$, consider the curve $I_{\delta}$ :

$$
\phi=\frac{\pi}{2}+\delta\left(\frac{1}{2}+\sin 2 n \theta\right)
$$

where $n$ is a positive integer and $\delta$ is a small positive constant. This curve cuts through the $\left(x_{1}, x_{2}\right)$-plane at $4 n$ points regularly distributed in pairs about the circle $\phi=\pi / 2$, and hence $O$ is interior to the convex hull of these points. A similar statement can then be made concerning the point $(0,0, \epsilon)$ with respect to the points in which the plane $x_{3}=\epsilon$ meets $I_{\delta}$, provided $\epsilon \lessgtr 0$ is sufficiently small in numerical value. Consequently, $O$ is interior to the convex hull of $I_{\delta}$.

Since $I_{\delta}$ is symmetric in the $x_{3}$-axis, its center of gravity lies on this axis. That $G$ does not coincide with $O$ is evident from the fact that the maximum value of $\phi$ is $\pi / 2+(3 / 2) \delta$, whereas the minimum value is $\pi / 2-\delta / 2$. Hence, a plane $\pi$ of the definition of a curve of type $A$ is in this case a horizontal plane, say, the $\left(x_{1}, x_{2}\right)$-plane. But, for $\delta$ sufficiently small, the projection of $I_{\delta}$ on the $\left(x_{1}, x_{2}\right)$-plane is certainly an oval, inasmuch as the curvature of the projection is a continuous function of $\delta$ and, when $\delta$ approaches zero, the projection approaches the circle $\phi=\pi / 2$. Therefore, the curve $I_{\delta}$, for sufficiently small values of $\delta$, is the tangent indicatrix of a twisted curve of type $A$.

An example of a curve on the unit sphere which is the tangent indicatrix of a twisted curve of type $A$ and has its center of gravity at $O$ is given by $\phi=\pi / 2+\delta \sin 2 n \theta$, where $n$ is a positive integer and $\delta$ is a positive constant, sufficiently small.

HARVARD UNIVERSITY

See also Fenchel, Geschlossene Raumkurven mit vorgeschriebenen Tangentenbild, Jahresbericht der Deutschen Mathematiker Vereinigung, vol. 39 (1930), pp. $183-185$. 